\title{
CONTRIBUTION OF SEDENTARISM TO ALZHEIMER'S DISEASE-RELATED DECLINE: A CROSS-SECTIONAL STUDY
}

Felipe de Oliveira Silva ${ }^{1}$, José Vinícius Ferreira ${ }^{1}$, Jessica Plácido ${ }^{1}$, Jerson Laks $^{2}$, Andrea Deslandes ${ }^{1}$

1. Federal University of Rio de Janeiro, UFRJ; 2. Grande Rio University, Unigranrio.

\section{BACKGROUND AND AIM}

Alzheimer's disease (AD) is a neurodegenerative disease associated to cognitive and physical declines. However, sedentary behavior also leads to a deterioration of global function.

\section{PURPOSE}

The purpose of this study was to compare cognitive and physical functions of sedentary and active elderly with AD.

\section{METHODS}

AD patients were separated according to the level of weekly physical activity (PA) recorded by the International Physical Activity Questionary (IPAQ: <600 met / week [Sedentary], >600 met / week [Active]) (Table 1). An independent t-test was performed to analyze the difference between groups and the effect size (ES) was used to compare the cognitive (RAVLT_A1A5 = memory test; TMTB = trail making test $B$; VF = verbal fluency) and physical functions (STS = sit to stand test; 8UG_ST = 8-foot up and go with single task; STEP = aerobic functional test) at different PA levels

\section{RESULTS}

There were statistical differences between groups in aerobic capacity and strength training (Table 2). The ES were large (total physical function ES= 1.21) and moderate (total cognitive functions $E S=0.56$ ) in favor of the active elderly compared to the sedentary ones (Figure $1)$.

Table 1 - Descriptive analysis of the sample (mean \pm SD)

\begin{tabular}{lcccc}
\multicolumn{1}{c}{ Features } & $\begin{array}{c}\text { Sedentary AD } \\
(\mathbf{n}=\mathbf{9})\end{array}$ & $\begin{array}{c}\text { Active AD } \\
(\mathbf{n}=16)\end{array}$ & $\mathbf{t} /{ }^{*} \mathbf{U}$ & $\mathbf{P}$ \\
\hline Age, $\mathbf{y}$ & $78.76 \pm 8.14$ & $78,63 \pm 6,90$ & 0.050 & 0.961 \\
Female gender, $\mathbf{n}(\%)$ & $6(67)$ & $8(50)$ & ${ }^{*} 0.040$ & 0.841 \\
Schooling, $\mathbf{y}$ & $8.44 \pm 3.81$ & $11,64 \pm 6,42$ & -1.344 & 0.193 \\
MMSE, score & $17.00(11.00-23.00)$ & $20,00(16,00-26,00)$ & ${ }^{*}-2.487$ & 0.073 \\
BMI, kg/m ${ }^{2}$ & $27.10 \pm 4.42$ & $25,00 \pm 3,66$ & 1.282 & 0.213 \\
Medications, $\mathbf{n}$ & $2.00(1.00-5.00)$ & $2,00(1,00-4,00)$ & ${ }^{*} 0.279$ & 0.890
\end{tabular}

Table 2 - Analysis of physical and cognitive tests in sedentary and active AD patients.

\begin{tabular}{|c|c|c|c|c|}
\hline & $\begin{array}{c}\text { Sedentary AD } \\
(n=9)\end{array}$ & $\begin{array}{c}\text { Active AD } \\
(n=16)\end{array}$ & $t /{ }^{*} U$ & p \\
\hline \multicolumn{5}{|c|}{ Physical tests (mean \pm SD). } \\
\hline STS, $n$ & $9.11 \pm 2.52$ & $11,63 \pm 2,66$ & -2.312 & $0.030 \#$ \\
\hline 8UG_ST, s & $9.60 \pm 4.07$ & $6,87 \pm 1,17$ & 1.858 & 0.102 \\
\hline STEP, $n$ & $40.89 \pm 17.45$ & $80,19 \pm 26,03$ & -4.029 & $0.001 \#$ \\
\hline \multicolumn{5}{|c|}{ Cognitive tests (mean $\pm S D$ ). } \\
\hline RAVLT_A1A5, n & $16.44 \pm 4.36$ & $18,67 \pm 7,03$ & -0.851 & 0.404 \\
\hline DSB, $n$ & $2.78 \pm 1.72$ & $3,67 \pm 1,80$ & -1.191 & 0.246 \\
\hline VF, $\mathbf{n}$ & $6.89 \pm 2.42$ & $9,87 \pm 4,00$ & -2.014 & $0.056 \#$ \\
\hline
\end{tabular}

$\mathrm{AD}=$ Alzheimer's Disease; STS = Sit to stand test; $8 \mathrm{UG}=8$-foot up and go; ST = single task; STEP = aerobic functional test RAVLT A1A5 = memory test; $\mathrm{DSB}=$ Digit Span Backward; $\mathrm{VF}=$ Verbal fluency; $\mathrm{t}=$ independent test; ${ }^{*} \bar{U}=$ Mann-Whitney $\mathrm{U}^{*}{ }^{*}$ median (minimum - maximum); $\# \mathrm{p} \leq 0.05$.

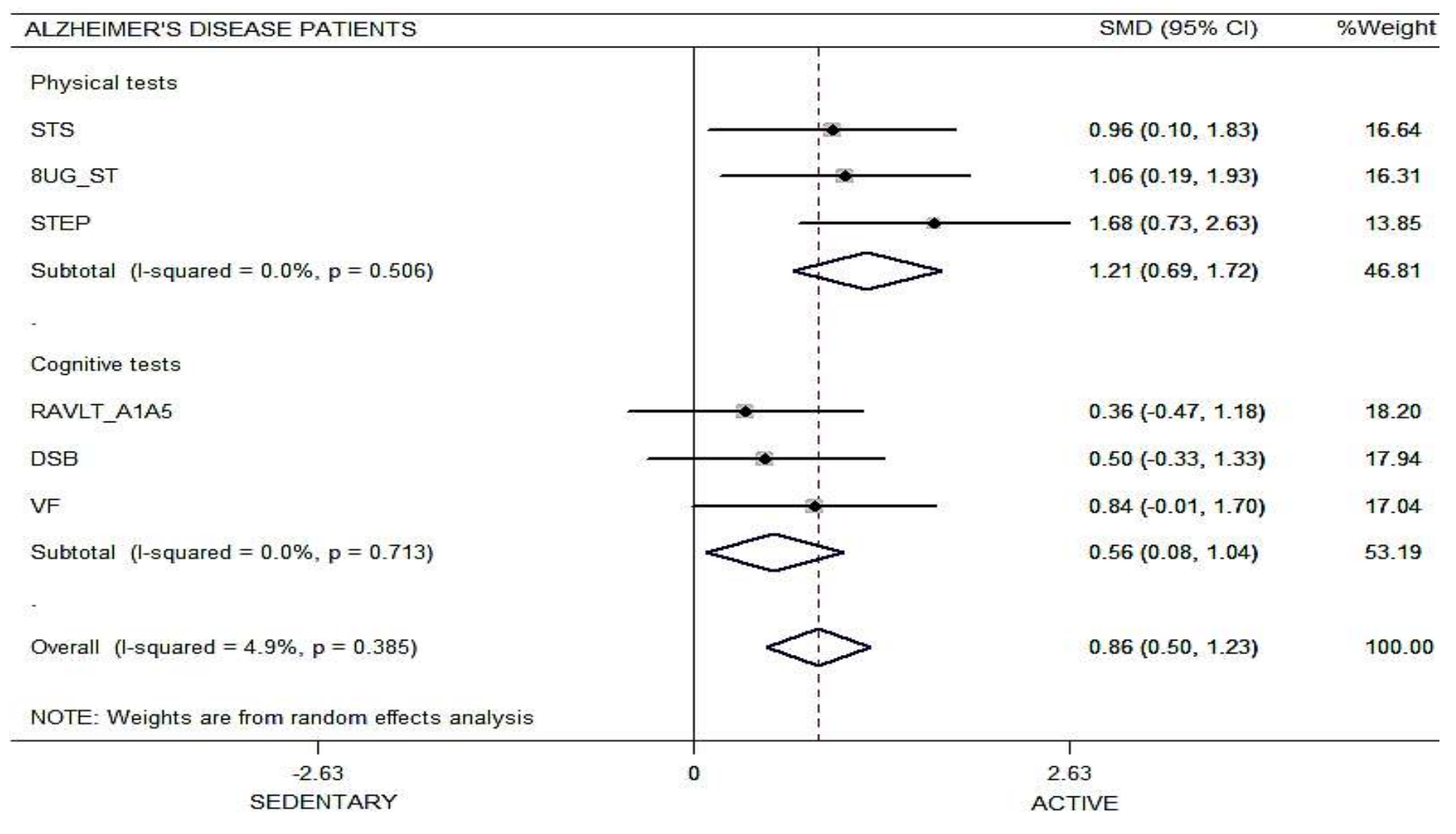

Figure 1 - Effect size of physical and cognitive tests in sedentary and active AD patients.

\section{CONCLUSION}

Moderate weekly physical activity can counteract disease-related decline in global functioning in patients with $A D$

\section{REFERENCE}

Liang, J.H., Xu, Y., Lin, L., Jia, R.X., Zhang, H.B., Hang, L., 2018. Comparison of multiple interventions for older adults with Alzheimer disease or mild cognitive impairment: $A$ PRISMA-compliant network meta-analysis. Medicine (Baltimore) 97;

Groot, C., Hooghiemstra, A.M., Raijmakers, P.G., van Berckel, B.N., Scheltens, P., Scherder, E.J., van der Flier, W.M., Ossenkoppele, R., 2016. The effect of physical activity on cognitive function in patients with dementia: A meta-analysis of randomized control trials. Ageing research reviews $25,13-23$.

\section{ACKNOWLEDGEMENT AND CONTACT}

prof.felipedeoliveira@gmail.com adeslandes@ipub.ufrj.br

10 @prof.felipedeoliveira @lanexufrj
Supported by $\mathrm{CNPq}$ (301483/2016-7) and FAPER (E-26/ 203.193/2016).

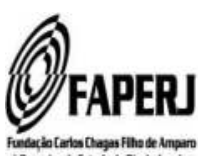

